\title{
Development of a Complex Network-Based Integrated Multilayer Urban Growth and Optimisation Model for an Efficient Urban Traffic Network
}

\author{
Rui Ding $\mathbb{D}^{1,2,3}$ Yilin Zhang, ${ }^{1,2,3}$ Ting Zhang $\mathbb{D}^{1,2,3}$ and Can $\mathrm{Ma}^{1,2,3}$ \\ ${ }^{1}$ School of Big Data Application and Economics (Guiyang College of Big Data Finance), \\ Guizhou University of Finance and Economics, Guiyang 550025, China \\ ${ }^{2}$ Guizhou Key Laboratory of Big Data Statistical Analysis, Guizhou University of Finance and Economics, Guiyang 550025, China \\ ${ }^{3}$ Key Laboratory of Green Fintech, Guizhou University of Finance and Economics, Guiyang 550025, China
}

Correspondence should be addressed to Ting Zhang; 269493818@qq.com

Received 7 June 2020; Revised 3 December 2020; Accepted 6 January 2021; Published 23 January 2021

Academic Editor: Jiaojiao Jiang

Copyright (c) 2021 Rui Ding et al. This is an open access article distributed under the Creative Commons Attribution License, which permits unrestricted use, distribution, and reproduction in any medium, provided the original work is properly cited.

\begin{abstract}
Previous research studies of traffic networks are mainly based on planar networks and less considered the influence of multilayer networks, which illustrate and represent different appropriate urban traffic modes. Development of rail and road networks is inseparable from the development of a prosperous urban area; thus, research on multilayer networks has scientific potential and fulfils a real need. In this paper, a framework of complex network based integrated multilayer urban growth and optimisation model (CNIMUGOM) is proposed, to analyse the complex relationships between the traffic network structure, the population growth, and the urban land-use. The innovation of this paper is the combination of the traffic complex multilayer networks and the "Four Step Model" (which stands for trip generation, trip distribution, model split, and traffic assignment steps). With the multiobjective, multilayer network coevolution and optimisation model, a more efficient traffic network layout was generated based on different land-use, population density, and travel speed scenarios. Then, this paper has proved that the proposed CNIMUGOM can save the traffic network construction investment, reduce the travel cost, make the urban traffic network more efficient, and decrease the total traffic flow amount. This research has connected the recent complex multilayer network related study and traditional urban economic model based study. The findings of the study afford to improve the current land-use and traffic integrated models and can provide traffic network planning suggestions for urban agglomeration development.
\end{abstract}

\section{Introduction}

Many developing countries still face rapid urbanisation process, with numerous scholars focusing on the prompt urban traffic network growth and coevolution process [1-3]. To measure the growth and coevolution process of an urban traffic network, research concentrated on two aspects: the first is modelling and simulation, and the second is its natural course [4-6]. The study of the coevolution process can clearly analyse the interrelationships between urban land-use and traffic networks $[7,8]$. Focusing on the coevolution model of the $19^{\text {th }}$ and $20^{\text {th }}$ century in London, the group of Levinson and Xie found that population distribution and network density are positively correlated. They validated a simulation model to fit the empirical evidence better and noted that evolution is an iterative process of interaction, investment, and divestment. Moreover, they illustrated how surface traffic networks could grow and decline spontaneously over time, providing further evidence for the property of self-organisation [5, 9-11]. Similarly, a new dynamic model based on the logistic equation, which captures the dynamic characteristics of the coevolution process between the street surface and urban traffic structure, was developed [12]. After that, the coevolution of urban traffic growth grounded in the case of Beijing is described [13], and a coevolution model is suggested with stability analysis and numerical simulation. In another way, Rui [14] used the multiagent based model to discuss the complex traffic network growth and land-use coevolution 
process, and centrality indices from different aspects were thoroughly analysed with the study of Stockholm. Recently, Li et al. [15] proposed an optimal urban expressway system model, which considered the interaction equilibrium of transportation and land-use.

Although those previous investigations are essential and necessary for further research of urban traffic network and land-use, they all treat networks as purely planar. Some of them considered the network growth and variation, but none of them considered the influence of multilayer networks, which is the cause of attention to multilayer networks related analysis.

Development of rail and street networks is inseparable from the development of a prosperous urban area; thus, research on multilayer networks has scientific potential and fulfils a real need. To bridge the gap of the multilayer network representation of real-world networks, Kurant and Thiran [16] first proposed a general multilayer model that facilitated the description and analysis of multilayer networks. The authors examined three transportation networks and found that a small error on a multilayer network could cause cascading failures. They also investigated the relationships between degree, betweenness, and real loads and found that, as opposed to the commonly acknowledged view, the correlations in their dataset between the three factors were not that apparent. More recently, multilayer networks earned more attention, as traffic dynamics in two-layer complex networks were considered by Ma et al. [17], and Albert et al. [18] introduced a standardised model to simulate the elements navigating those networks and analysed congestion in multilayer transportation networks. Furthermore, the ratio of speeds of coupling different modes, network accessibility, mobility, and behaviour of different layers were considered [18-23]. These researches bring a new broad of perspective, investigating the aspects of multilayer networks to consider the interrelationship and cooperation of different traffic layers and modes [22, 23].

With the novel study of multilayer networks, the relationships between the urban traffic networks and land-use of surrounding areas might be discussed. With the analysis of some new indicators, their complex relationships become measurable. This fills the research gap of recent urban traffic network structure based studies; with the study of network coevolution process, the complex dynamics growth process can be partly studied. The influence of multilayer networks can be also measured, which connect the growth of upperlayer and lower-layer network and the accessibility change of surrounding areas related to urban land-use [24]. Based on these works, in this paper, the framework of complex network based integrated multilayer urban growth and optimisation model (CNIMUGOM) will be proposed first. Considering the traffic network structure, with the population growth rate rp and its affection of urban land-use model (the change of accessibility of employment $A_{i}^{E}$ and population $A_{i}^{P}$ ), and the coupling features of multilayer networks, the study identifies issues regarding the "Four Step Model" (FSM). For the travel demand model, the number of trips generated $O_{i}$ and attracted $D_{i}$; the number of trips from traffic zone $i$ to zone $j$, $T_{i j}$; the generalised travel cost $t_{i j}$; the travel time on the link $a$, $t_{a}$; and the traffic flow $f_{a}$ will be calculated. For the street investment model and traffic network growth model, the collected revenue of this link $R_{a}$, the measurement of multilayer network structure status $\mathrm{NS}_{i}$, the overall spending function $S_{a}$, and the general investment $I^{k+1}$ of iteration $k+1$ will be calculated in Matlab. With the multiobjective, multilayer network coevolution and optimisation model, the more efficient network layout can be generated based on some different land-use (different population density) scenarios. Based on the traffic network and urban land-use coevolution process, the proposed CNIMUGOM can save the traffic network construction investment, reduce the travel cost, and make the urban traffic network more efficient. Based on the simulation, the proposed network can increase network efficiency and decrease the total traffic flow amount.

\section{Methodology}

This study refers to the general urban transportation system as a multilayer network. The upper-layer network represents the public rail network, which may include rapid transit, LRT, monorail, MRT, and subway. Here, this study does not distinguish between rail transit modes. The lower-layer network represents urban street and road networks.

2.1. Single-Layer Network Representation Method. This method is used by many scholars, and related studies of different cities were widely accepted [25-28]. According to the primal approach, the single-layer network representation method, as shown in Figure 1, the black lines on the right act as streets or roads, the nodes represent the street intersections, and the grey spots are buildings.

With this method, the different undirected or directed networks can be used to represent the urban transportation networks:

$$
G=\langle V, E, W\rangle,
$$

where $V$ is the set of nodes, $N$ is the number of nodes when

$$
V=\left\{v_{i} \mid i \in I \equiv\{1,2, \ldots, N\}\right\},
$$

$E$ is the unordered pairs or edges of elements of $V$ and is denoted by $e_{i j}$,

$$
E=\left\{e_{i j}=\left(v_{i}, v_{j}\right) \mid i, j \in I\right\},
$$

and $W$ is the weight of each edge; in some functions, it can be denoted as $w$, and the weight can be treated as the length of the edge or lanes.

The number of edges is denoted as $M$. The adjacency matrix of networks is

$$
A=\left[a_{i j}\right]_{n \times n}
$$

representing the connection between nodes $v_{i}$ and $v_{j}$, which is defined as

$$
a_{i j}= \begin{cases}1, & \left(v_{i}, v_{j}\right) \in V, \\ 0, & \left(v_{i}, v_{j}\right) \notin V,\end{cases}
$$

where $a_{i i}=0$ to remove any self-connections. In addition, $A=\left[a_{i j}\right]_{n \times n}$ is symmetrical and nonnegative. 


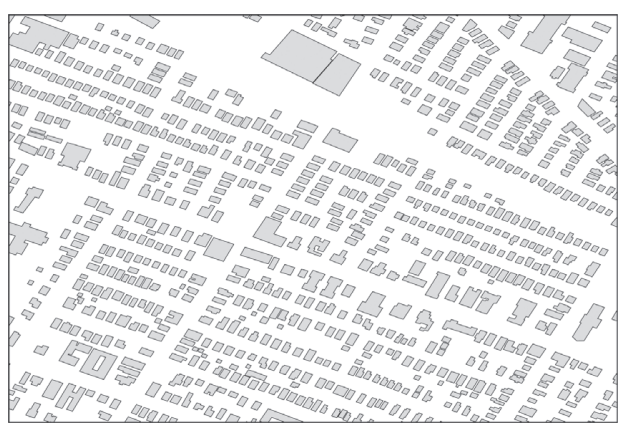

(a)

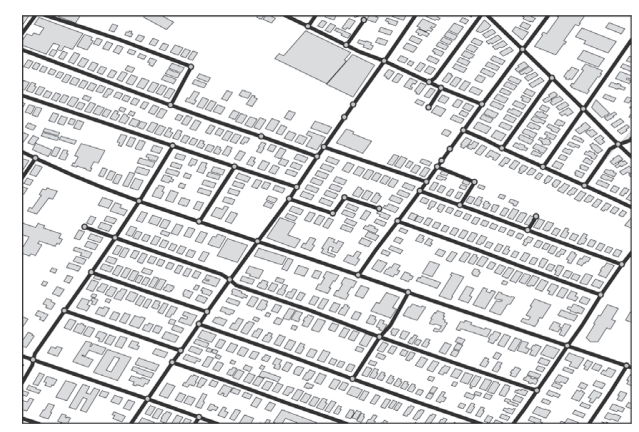

(b)

FIgURE 1: The single-layer network representation method.

We define $D$ as the network diameter, with

$$
D=\max \left\{S_{i j}\right\}, \quad 1 \leq D \leq(N-1) .
$$
and $j$.

Here, $S_{i j}$ is the sum of edge numbers between node pair $i$

The degree centrality was proposed by Freeman [29]; the function is

$$
\mathrm{DC}\left(v_{i}\right)=\frac{k_{i}}{N-1} \text {. }
$$

The number of link incidents on a node can reflect the importance of the node $v_{i}$ in relation to spatial geography, which indicates that a node with more neighbours is more important in a network.

The closeness centrality was proposed by Marchiori and Latora [30]; the function is

$$
C_{\text {closeness }}\left(v_{i}\right)=\frac{1}{D_{i j}}=\frac{N-1}{\sum_{i \neq j} d_{i j}} .
$$

It is denoted as the reciprocal of the average distance between each node pair $D_{i j}=\left(\sum_{i \neq j} d_{i j} / N-1\right)$. This index means that if a node is closer to the other remaining nodes, it is more important in the network; it describes the relative location of a node.

The betweenness centrality was proposed by Freeman [29]; the function is

$$
\mathrm{BC}\left(v_{i}\right)=\frac{\sum_{i \neq s \neq t \in V}\left(d_{\text {min }, \mathrm{st}}^{i} / d_{\min , \mathrm{st}}\right)}{(N-1)(N-2)} .
$$

It is defined as the total number of shortest paths between two separate nodes $d_{\min \text {,st }}$ and passing through node $v_{i}$; it reflects the load on node $v_{i}$ and can be alternately understood as the controllability of the node. On this basis, centrality can be clarified as

$$
C_{\text {betweenness }}\left(v_{i}\right)=\sum_{i \neq s \neq t \in V} \frac{d_{\min , \mathrm{st}}^{i}}{d_{\min , \mathrm{st}}},
$$

and the normalisation of betweenness $\mathrm{BC}\left(v_{i}\right)$ is described as the function shows, where $(N-1)(N-2)$ is the maximum possible value.

The average shortest path length was proposed by Albert and Barabási [31]; the function is

$$
\mathrm{APL}=\frac{1}{N(N-1)} \sum_{i \neq j} d_{\min }^{i j} .
$$

It is defined as the average number of steps along the shortest paths for all possible pairs of network nodes.

The network efficiency $E(G)$ was proposed by [32]; the function is

$$
E(G)=\frac{1}{N(N-1)} \sum_{i \neq j} \frac{1}{d_{\min }^{i j}} .
$$

It shows the average efficiency of transit flow or information between nodes in the network.

2.2. Multilayer Network Representation Method. Gu et al. [33] used this method to study airline networks and rail networks. The formula of the undirected multilayer network (see Figure 2) can be represented as

$$
G=G^{U}, G^{L},
$$

as the set of different layers; here, the superscript $U$ is used to define the upper-layer network and superscript $L$ to set the lower-layer [33, 34].

The connected network can be used to represent the rail network and urban street network as

$$
\begin{aligned}
& G^{U}=V^{U}, E^{U}, W^{U}, \\
& G^{L}=V^{L}, E^{L}, W^{L} .
\end{aligned}
$$

The weight is denoted by $W$ in the function [35]. Red nodes represent rail stations, and blue nodes represent street intersections; solid lines represent their connections, and dotted lines represent cross links (cooperative relationship) between different layers. The rail network station is connecting with the nearest street network intersection and its weight is equal to 1 [23]. To simplify the transfer process, when measuring the accessibility, this study sets the transfer time between the two layers to 3 minutes [36]. The multimodal transportation and relations between the vehiclebased network and the pedestrian network can be gained from the multilayer network.

The multilayer network model of urban traffic networks; the upper-layer represents rail network topology, and the 


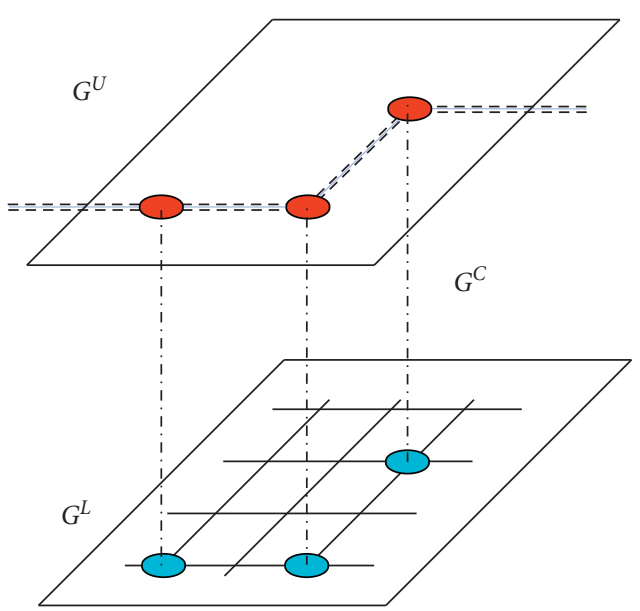

FIgURE 2: The multilayer network representation method.

lower-layer represents the street network topology or OD zones.

In this definition, $V$ is the set of the street network topology, and $\mathrm{N}$ is the node number of lower-layer when

$$
V=\left\{V_{i} \mid i \in I \equiv\{1,2, \ldots, N\}\right\},
$$

and $E$ represents the unordered pairs of edges and elements of $V$ and is denoted by $e_{i j}$

$$
E=\left\{e_{i j}=\left(v_{i}, v_{j}\right) \mid i, j \in I\right\} .
$$

Similarly, the definition of the multilayer network is

$$
\begin{aligned}
& N^{\text {multi }}=N^{U}+N^{L}, \\
& V^{\text {multi }}=V^{U}+V^{L}, \\
& E^{\text {multi }}=E^{U}+E^{L}+E^{C} .
\end{aligned}
$$

The adjacency matrix of networks adj $=\left[a_{i j}\right]_{n \times n}$ is symmetrical and nonnegative, representing the connection between zones $i$ and $j$, where

$$
a_{i j}= \begin{cases}d_{i j} \times W, & \left(v_{i}, v_{j}\right) \in E, \\ 0, & \left(v_{i}, v_{j}\right) \notin E,\end{cases}
$$

where $d_{i j}$ is the Euclidean distance. Define $a_{i i}=0$ to theoretically remove any self-connections to exclude the impact of the network element itself. Then, the adjacency matrix of multilayer networks is

$$
\operatorname{adj}^{\text {multi }}=\left[\begin{array}{ll}
\operatorname{adj}_{N^{U} \times N^{U}}^{U} & \operatorname{adj}_{N^{U} \times N^{L}}^{C} \\
\operatorname{adj}_{N^{L} \times N^{U}}^{C} & \operatorname{adj}_{N^{L} \times N^{L}}^{L}
\end{array}\right] .
$$

\subsection{Analysing the Coevolution Process of Multilayer Network}

2.3.1. The Inner Connections of Multilayer Networks. For a big city, building all of these traffic links as railways is a waste of money and less effective, and only constructing streets without any massive rapid transit will cause a heavy traffic jam. The specific structure of the traffic multilayer networks is the result of the combination of natural and historical conditions, location characteristics, economic development and conditions, urban space layout, and even traffic customers. The network structures determine the relationships of different layers; they are highly related but have apparent differences. One of them is the complementary element of another, using their advantages to make up for the deficiency of others. The structural change of one layer will cause the change of another layer. Their combination completes the urban traffic networks functions

The traffic distribution is more focused on the choice of routes between origin-destination OD zones. It is an iterated process which compares the trips assigned with the link capacity to find an equilibrium status and show the number of travellers on each route and link in a given transportation network. The classic User Equilibrium (UE) is widely approved and used to describe the user's route behaviour. Once the users believe that they find the smallest impedance (perceptual impedance), they will not change their routes, although the routes are not the smallest route and the impedance may not be the smallest route impedance. Thus, an equilibrium is reached. Related functions will be provided in later sections.

With the population movement and growth, the growth of upper-layer and lower-layer networks is determined; considering the UE problem, we use the Frank-Wolfe BPR method and set the travel cost on the congested links as infinite. The Frank-Wolfe algorithm is "an iterative firstorder optimisation algorithm for constrained convex optimisation" $[37,38]$, which is widely used to deal with the traffic equilibrium problem such as UE. After that, the network structure data, the cost of each link, the OD demand data, and the capacity of each link are obtained; then, we initialise the optimisation aims, construct the impedance function, and find the initial impedance. Then, we use the All or Nothing Assignment technique [39] to assign the traffic flow and update the data, and finally the optimal function value converging to $5 \%$ of estimation error range can be obtained. This technique is widely used and well accepted by many traffic network researchers [38, 40-42].

2.3.2. The Coevolution Relationships between Traffic Multilayer Network and Urban Land-Use. Many related types of research have discussed their connections and inner relationships [43, 44]. Because the traffic capacity and travel speed are different, the impacts of network structure change to land-use typically can be measured by the accessibility $[45,46]$. The spatial connection means that urban land-use growth is characterised by continuity in a planar space [47], and then it is meaningful for the measurement of the population diffusion and urban growth process. Urban landuse change process also has a positive correlation with the variation of the population [48].

Their coevolution relationships can be simply represented in Figure 3, in which the traffic generation and attraction are determined by the urban land-use spatial distribution; with the increase of population density, landuse intensity, and sprawl or generation of new land-use 


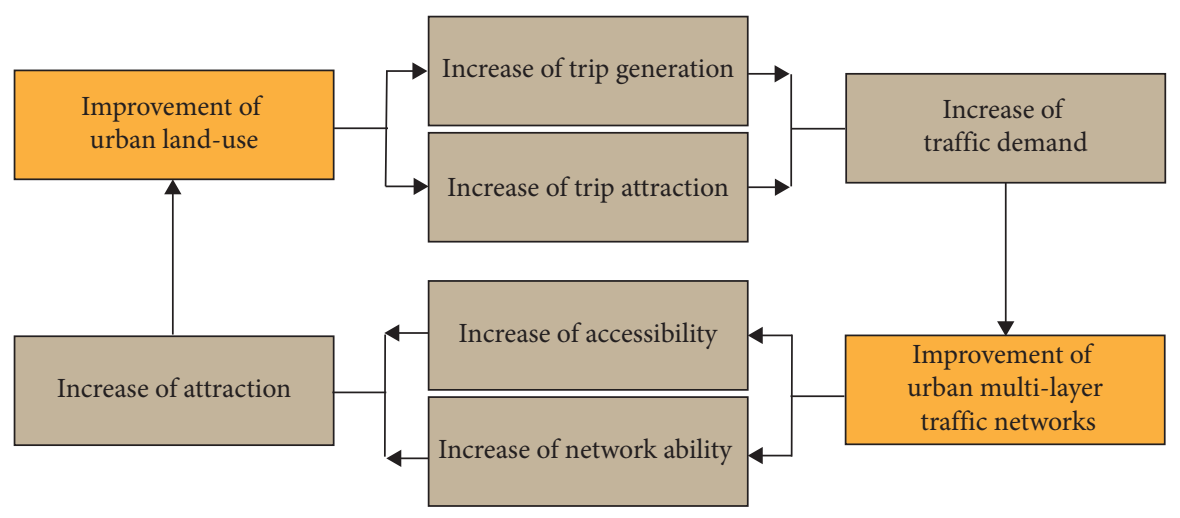

FIgURE 3: The simple coevolution relationships between traffic multilayer network and urban land-use.

parcels, traffic demand is increasing with different scales, which naturally needs more traffic infrastructures to satisfy it. In turn, the increase of the investment of multilayer traffic networks, which make the traffic networks grow densely, increases the accessibility of land parcels; as more streets pass the area, people can easily access this previously less developed area. The abilities of networks can also be improved, as the investment is always used to increase the capacities and the topological network functions of each layer. Based on the location theory and the scarcity of urban land resources, the attraction of these land parcels connected with traffic networks will increase. Then, more people are attracted, and the coevolution process emerges. Here, the land-use coevolution model is developed to reflect the relationship of accessibility with population growth and movement, and traffic network growth. Other factors, such as house price and related policies, are excluded to keep this relationship succinct and clear. Hence, the land-use model is highly simplified, mainly focused on the accessibility, and represented as the distribution of population and employment.

To represent the competition characteristic of neighbourhood interactions, the land-use coevolution model also includes both centrifugal and centripetal forces [49, 50]. Assume that people always want to live near to the location of jobs to save travel cost, but far away from other people such as potential contestants. However, on the other hand, employment wants to be accessible both to other businesses (to save cargo transportation cost) and to people (who are the suppliers of labour and customers). Both of them want to live with a higher traffic network structure service grade. Related functions will be provided in Section 3.

\section{The CNIMUGOM}

3.1. The Framework of CNIMUGOM. The content of the model is suggested, as shown in Figure 4, and different blocks stand for different models, with the relationships between those models illustrated as arrows. The most famous models are the travel demand model, street investment model, traffic network growth model, population growth model, urban land-use growth model, and network optimisation model.
3.2. Traffic Demand Model. The demographic, socioeconomic, land-use, and online hailing data were incorporated to reveal the root causing that influence traffic demand [51-53]. When considering the traffic network growth, an important part that must be focused on is that this kind of growth is always constrained and stimulated by traffic demand. Based on the initial traffic network data, the classic "Four Step Model" (FSM) can be generated and used, considering the multilayer networks and the network coevolution process. The travel demand model in an initial network is predetermined by population and employment data and related to the topology of the multilayer network.

3.2.1. Trip Generation Model. A simple traffic generation model was given by Levinson and Zhu [49] and Xie and Levinson [54], where trip generation and trip attraction of different traffic zones were calculated; with respect to the population and employment and their natural carriers, households and companies, the simple linear relationships were proposed, and the assumption formula is

$$
\begin{aligned}
& O_{i}=\xi_{0}+\xi_{1} E_{i}+\xi_{2} P_{i}, \\
& D_{i}=\psi_{0}+\psi_{1} E_{i}+\psi_{2} P_{i},
\end{aligned}
$$

where $O_{i}$ and $D_{i}$ stand for the number of trips generated and attracted; $E$ and $P$ stand for the amount of the employment and population of traffic zone $i$, respectively; and $\xi_{0}, \xi_{1}, \xi_{2}$, $\psi_{0}, \psi_{1}$, and $\psi_{2}$ are adjustable coefficients of this simple linear equation set, and their values are $0,0.5,1,0,1$, and 0.5 , respectively.

3.2.2. Trip Distribution Model. For the general distribution process, the mostly used doubly constrained trip distribution model is adopted, that is, a gravity-based model, to match both trip generation and attraction of locations based on a negative exponential function that assumes that the interactions of zones decrease with the travel time between them $[50,54]$ :

$$
T_{i j}=\left(K_{i} K_{j}\right) O_{i} D_{j} e^{-\phi t_{i j}}=\frac{O_{i} D_{j} e^{-\phi t_{i j}}}{\left(\sum_{i} K_{i} O_{i} e^{-\phi t_{i j}} \times \sum_{j} K_{j} D_{j} e^{-\phi t_{i j}}\right)},
$$




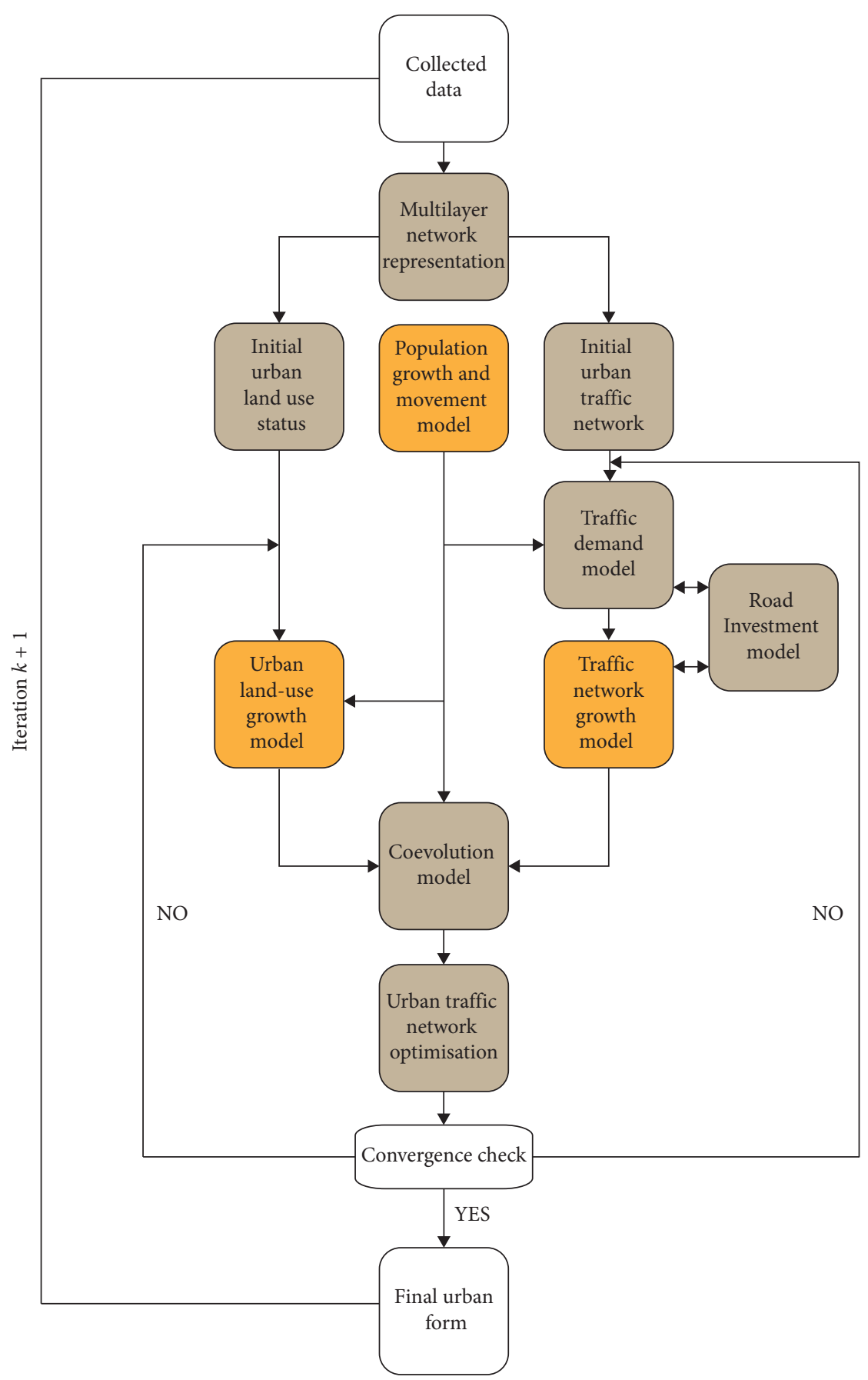

Figure 4: The framework of CNIMUGOM.

where $T_{i j}$ is the number of trips from zone $i$ to zone $j$, and its table can be gained as the OD matrix; $O_{i}$ is the number of trips attracted in zone $i ; D_{j}$ is the number of trips attracted by zone $j$; and $t_{i j}$ is the travel cost. $\left(K_{i} K_{j}\right)=\left(1 /\left(\sum_{i} K_{i} O_{i}\right.\right.$ $\left.\left.e^{-\phi t_{i j}} \times \sum_{j} K_{j} D_{j} e^{-\phi t_{i j}}\right)\right)$ are the balancing coefficients which were precalibrated $[55,56]$.

3.2.3. Measurement of the Network Structure Related to Generalised Travel Cost. The generalised travel cost as normal travel cost $t_{i j}$ is measured by travel time, a generalised concept considering the congested travel cost, zonal accessibility, and so forth from zone $i$ to zone $j$ and considering the intrazonal and interzonal costs. Hence, the equation can be written as

$$
t_{i j}= \begin{cases}\sum_{a}\left(\delta_{i, j}^{a}, t_{a}\right)+t_{m, i}+t_{m, j}, & \text { for } i \neq j, \\ t_{m, i}, & \text { for } i=j,\end{cases}
$$

where $\sum_{a}\left(\delta_{i, j}^{a}, t_{a}\right)$ is the interzonal travel time which is the summation of travel costs along the shortest path between $i$ and $j$, and $t_{a}$ represents the generalised travel time that vehicle spends on the link $a$; if this link exactly belongs to the 
shortest path between zone $i$ and zone $j, \delta_{i, j}^{a}=1$; otherwise, $\delta_{i, j}^{a}=0$. The $t_{m, i}$ embodies the generalised intrazonal travel time in zone $i$; this is a simplified measure method also for the convenient calculation. Sometimes, the intrazonal costs are neglected for convenient calculation, but they will be counted especially when the zone has higher land-use intensity and density; they capture a variety of costs incurred on trips associated with land-use and traffic network structure differences.

The travel time on the link $a$ is measured as $t_{a}$; normally, it can be calculated as the ratio between the street length $l_{a}$ and the speed $v_{a}$; however, it is always constrained by the traffic flow $f_{a}$ and the collected revenue of this link $R_{a}$ :

$$
t_{a}=\frac{l_{a}}{v_{a}}+\frac{R_{a} / \eta}{f_{a}} .
$$

Here, $\eta$ is the balance parameter, which represents the average value of time.

The BPR function is used to reflect the relationship of the free flow time $v_{a}^{f}$ and congested speed $v_{a}^{c}$ on link $a, C_{a}$ is the link capacity, and $\alpha_{\mathrm{BPR}}$ and $\beta_{\mathrm{BPR}}$ are correction factors equal to 0.15 and 4 , respectively:

$$
v_{a}^{c}=\frac{v_{a}^{f}}{\left[1+\alpha_{\mathrm{BPR}}\left(f_{a} / C_{a}\right)^{\beta_{\mathrm{BPR}}}\right]} .
$$

For example, in a zone with higher land-use intensity and density, maybe the jobs provided and population amount are much more than the average level; thus, the travel cost will increase correspondingly considering the traffic congestion levels, longer elevator wait time in skyscrapers, and greater difficulty of finding parking. On the other hand, influenced by the traffic network structure, within most of the situations (without considering Braess's paradox), with higher traffic network structure service level, it will alleviate the traffic congestion and increase the accessibility, and usually the cost will be reduced. Based on these assumptions it can be measured as

$$
t_{m, i}=\chi t_{m}^{0} \frac{\left[1+\left(G_{i} / \bar{G}\right)^{2}\right]}{\left[1+\left(\mathrm{NS}_{i} / \overline{\mathrm{NS}}\right)^{2}\right]},
$$

where $t_{m}^{0}$ is a specified base intrazonal travel cost for all zones, $\chi$ is a changeable parameter to balance the impact of network structure, and $\chi=1 . G_{i}$ is the number of activities (the employment and population) in zone $i$; the function is $G_{i}=E_{i}+P_{i}$, connecting with the trip generation model. $\bar{G}$ is the average number of activities for all zones. $\mathrm{NS}_{i}$ is the measurement of network structure status; generally for a zone with higher traffic infrastructure level, the time used to pass this zone will lower; hence, considering those fundamental traffic network indicators and MCA indicators, this study chooses the node degree centrality DC, the betweenness $C_{\text {betweenness }}$, and the closeness centrality $C_{\text {closeness }}$ as related indicators because of the previous analysis, and the function is

$$
\mathrm{NS}_{i}=\varepsilon_{\mathrm{DC}} \mathrm{DC}+\varepsilon_{\mathrm{BC}} C_{\text {betweenness }}+\varepsilon_{\mathrm{CC}} C_{\text {closeness }},
$$

where $\varepsilon_{\mathrm{DC}}, \varepsilon_{\mathrm{BC}}$, and $\varepsilon_{\mathrm{CC}}$ are parameters equal to 1000,1 , and 10000 , respectively, and $\overline{\mathrm{NS}}$ is the average status of network structure for all zones.

3.2.4. Mode Choice in a Multilayer Network. In this research, mode choice is used to connect the travel demand with multilayer networks. Over this process, the trips between a given origin and destination are split into trips using transit and automobile for simplicity. The most frequently used function is Binary Logit Model [57], also called "econometric formulation," which merely refers a log ratio of the possibility of choosing a mode $\left(\mathrm{Po}_{i}\right)$ to the possibility of not choosing this mode $\left(1-P o_{i}\right)$, and the function is

$$
\begin{aligned}
\log \left(\frac{P o_{i}}{1-P o_{i}}\right)= & v\left(x_{i}\right)=\beta_{0}+\beta_{1}\left(c_{A}-c_{R}\right)+\beta_{2}\left(t_{A}-t_{R}\right) \\
& +\beta_{3} I_{c}+\beta_{4} N_{t},
\end{aligned}
$$

where $\beta_{0,1,2,3,4}$ are related parameters, $c_{A}$ and $c_{R}$ are the travel costs of using the mode of street and rail transit (the measurement of $c_{A}$ only considers the situation of travel using the street system, but $c_{R}$ considers using street and rail systems and the cooperation parts), $t_{A}$ and $t_{R}$ are the travel times for using the mode of street and rail transit, $I_{c}$ stands for income, and $N_{t}$ is the number of travellers. This function has deterministic meaning; that is, when faced with the same options, they will always make the same choices. Hereby considering the multilayer relationship, a function set is proposed; related parameters refer to the basic multilayer network indicators, combined with the BPR function and the real traffic loads on the link $a$. This function set is

$$
\begin{aligned}
& c_{A}=\left(w_{s} \tau_{s}\right) l_{a} \sum f_{a}, \\
& c_{R}=\left(w_{s} \tau_{s}\right) l_{a} \sum f_{a}+\left(w_{c} \tau_{c}\right) l_{c}+\left(w_{r} \tau_{r}\right) l_{r} \sum f_{r}, \\
& t_{A}=\frac{l_{a}}{v_{a}^{f}\left[1+\alpha\left(f_{a} / C_{a}\right)^{\beta}\right]}+\frac{R_{a} / \eta}{f_{a}}, \\
& t_{R}=\frac{l_{r}}{v_{r}}+\frac{R_{r} / \eta}{f_{r}},
\end{aligned}
$$

where $l_{a}$ and $l_{r}$ are the length of automobile and train transit routes, $v_{r}$ is the average speed of rail transit, $f_{r}$ is the rail traffic flow, and $R_{r}$ is the collected revenue of this rail link. The part $\left(\left(R_{r} / \eta\right) / f_{r}\right)$ also means the transfer and wait time cost; for a higher investment rail line, the transfer and wait time are usually smaller.

3.2.5. Traffic Assignment Model. Here, User Equilibrium (UE) model is chosen; this model is proposed by Sheffi [42] and has been well recognised and validated by the traffic researchers; here, it will not be discussed further, and these conditions can be referred to in Sheffi [42]. 


$$
\begin{aligned}
& \max E(G), \\
& \min \quad C_{\text {system }}=\sum x_{a} t_{A} \\
& \text { S.T. }\left\{\begin{array}{l}
l_{a}, x_{a} \geq 0, \\
\sum\left(f_{a}\right)_{i j}=T_{i j}, \forall i, j .
\end{array}\right.
\end{aligned}
$$

Then, the lower-level function is shown as

$$
\begin{aligned}
\min Z(x) & =\sum_{a} \int_{0}^{x_{a}} t_{a}\left(x_{a}\right) \mathrm{d} x \sum_{p} f_{p}^{i, j}=q_{i j}, \forall i, j, \\
x_{a} & =\sum_{i} \sum_{j} \sum_{p} \delta_{a, p}^{i, j} f_{p}^{i, j}, \forall a, \\
\text { S.T. } \quad f_{a}^{i, j} & \geq 0, \forall p, i, j, \\
x_{a} & \geq 0, a \in S_{\text {link }} .
\end{aligned}
$$

Here, $x_{a}$ is the equilibrium flows of link $a, t_{a}$ is travel time, and the function is purposed to get the lowest travel costs. The $p$ is the path, $f_{p}^{i, j}$ is the traffic flow of OD pair $i$ and $j, q_{i j}$ is the trips between $i$ and $j, \delta_{i, j}^{a}$ is a definitional constraint, and $S_{\text {link }}$ is the set of links in the network.

3.3. Link Investment Model for Multilayer Network. The network growth process from the network structure parts is discussed previously, but in fact, this process is constrained by the investment inputs too. As mentioned before, this study is mainly considering a close network system; here, this study also treats the growth process as autonomous investment process. The link investment models are following the definition of Levinson et al. [49] and Xie and Levinson [11], where they describe the economic decisions of individual links autonomously; that is, the price for using the street during a given period, which users should pay for this autonomous agent, is dependent on the general traffic flow and the total length of these links. After a period, all the revenues are used to improve the network structure and link capacity. In each period, it is assumed that the collected revenues of toll from links by a general toll rate $\iota 1=0.1$, and this is an iteration process:

$$
R_{a}^{k+1}=\iota 1 \cdot l_{a}^{k} \cdot\left(f_{a-}^{k}+f_{a+}^{k}\right)=\iota 1 \cdot l_{a}^{k} \cdot\left(2 f_{a}^{k}\right),
$$

where if the study treats the network as a directed graph, and the total traffic flow in a given period $k$ can be given as $f_{a-}^{k}$ and $f_{a+}^{k}$, or for an undirected graph as $2 f_{a}^{k}$, then the connection between the collected revenues and the travel time on the link $a$ can be discussed; that is, $t_{a}$ is determined by the $R_{a}^{k-1} . l_{a}^{k}$ is the link length of iteration $k$.

On the other hand, the maintenance cost function is based on the toll function; considering the link capacity $C_{a}^{k}$ and average speed $v_{a}^{k}, 12=0.0001$, for an ordinary street or rail line, the capacities in two directions are normally equal to each other, and the overall spending function $S_{a}$ is

$$
S_{a}^{k+1}=12 \cdot l_{a}^{k}\left(f_{a-}^{k}+f_{a+}^{k}\right) C_{a}^{k} v_{a}^{k}=12 \cdot l_{a}^{k}\left(2 f_{a}^{k}\right) C_{a}^{k} v_{a}^{k} .
$$

The function of this profit part is

$$
P^{k}=\sum R_{a}^{k}-\sum S_{a}^{k}=I^{k+1} \text {. }
$$

Here, it is assumed that $P^{k}$ is the profit of iteration $k$, also equal to the general investment $I^{k+1}$ of iteration $k+1$, then connect with the network growth models and network optimisation models in the next sections.

The investment model considers the general revenues and expenditure of the system. Here, as per these previously proposed models, if the gathered revenue exceeds the maintenance cost, the profit part will be spent at the end of a time period without saving it for the future, with all being used for the capacity increase of each line, with the function

$$
C_{a}^{k+1}=C_{a}^{k}\left(\frac{R_{a}^{k}}{S_{a}^{k}}\right)^{\rho},
$$

where $\rho=0.1$. The free flow speed of new links with new capacity can be measured by a log-linear relationship calibrated by Zhang and Levinson [58] as $v_{a}^{f}=-30.6+$ $9.8 \times \ln \left(C_{a}\right)$.

3.4. The Population Growth Model. The coevolution model has illustrated some basic points of the population movements and their impacts; they will be further discussed here. When dealing with the urban expansion process, especially the land-use change process and the evolution of the urban street network, the population is treated as the most frequently considered mechanism and has the most significant consequence [59]. Typically, the population evolution model includes two parts: the population movement and the natural increase. The natural increase of population will change the amount of the total population, whereas the population movement will change the population distribution [60].

For simplicity, it can be assumed that the natural increase rate of the population is a constant, and then a simple exponential function can be limitedly used, as follows:

$$
\frac{d P o p^{k+1}}{d t}=r p * P o p^{k}
$$

where $\left(d P o p^{k+1} / \mathrm{d} t\right)$ is the population increment of iteration $k+1, \operatorname{Pop}^{k}$ is the population amount of iteration $k$, and $\mathrm{rp}$ is the increase rate, as $\mathrm{rp}=0.015$ per iteration [61].

3.5. Urban Land-Use Change Model. For the evolution models, inspired by the research of Levinson et al. [49] and Xie and Levinson [11], accessibility $A$ was used to reflect the desirability of a land parcel by calculating the accessibility of opportunities and activities [62], showing that smaller opportunities provide diminishing influences, also called gravity-based measurement, assuming that it fits a negative exponential cost function:

$$
A_{i}=\sum_{j=1}^{J} D_{j} e^{-\vartheta c_{i j}},
$$


where $A_{i}$ is the accessibility of node $i$ to all opportunities $D_{j}$ in node $j$ if the total number of traffic analysis nodes is $J$. The $\vartheta$ is the travel cost sensitivity parameter $(\vartheta=0.048)$ to the node which also indicates how the accessibility of a node declines with the increase of travel time, and $c_{i j}$ is the travel cost from $i$ to $j$.

The only factors which affect location choice of households and companies are accessibility and population density. Then, for the accessibility of employment and population in a land parcel, the function can be expanded as

$$
\begin{aligned}
& A_{i}^{E}=\sum_{j=1}^{J} E_{j} e^{-\vartheta c_{i j},} \\
& A_{i}^{P}=\sum_{j=1}^{J} P_{j} e^{-\vartheta c_{i j}},
\end{aligned}
$$

where $A_{i}^{E}$ is the accessibility to employment from node $i$ while $A_{i}^{P}$ is the accessibility to population. In the multilayer model, $c_{i j}=c_{A}+c_{R}$; hence, the accessibility considered different travel modes. This potential measure method has its specific practical advantage; that is, it can be easily calculated by the existing land-use and transport data.

3.6. Network Structure, Land-Use, and Population Coevolution Model. The desirability function sets are developed to illustrate the dynamics of employment accessibility and population accessibility based on independent decisions concerning their locations and network structure service grade $N S_{i}$. For the people desirability, the parameters $\lambda_{1}, \lambda_{2}, \lambda_{3}$ are all equal to 1 :

$$
U_{i, P}=A_{i}^{E \lambda_{1}} A_{i}^{P \lambda_{2}} N S_{i}^{\lambda_{3}},
$$

and, for the dynamics of employment desirability, $\lambda_{4}, \lambda_{5}, \lambda_{6}$ are equal to $0.9,-0.9$ and 0.9 , respectively:

$$
U_{i, E}=A_{i}^{E \lambda_{4}} A_{i}^{P \lambda_{5}} N S_{i}^{\lambda_{6}},
$$

where, for the independent decisions made by people, the competition between employment accessibility and population accessibility acted as centrifugal forces. Conversely, for the independent decisions made by businesses, the employment accessibility and population accessibility both strengthen the employment desirability and serve as centripetal forces. $\lambda_{1}, \ldots, \lambda_{6}$ are the related parameters, and these relationships between parameters were discussed in Xie and Levinson [10] as follows: “. . . accessibility to jobs did show a statistically significant positive effect on home sale values, while accessibility to resident workers did show a statistically significant negative effect ..." (p.164).

The increase in employment and population in zone $i$ is proportional to the single node's desirability difference quantity; here, $\overline{U_{P}^{k}}$ and $U_{E}^{k}$ are the average desirability at iteration $k$, considering the natural increase rate $r$, and $\lambda_{7}=\lambda_{8}=1$; then, we have the function

$$
\begin{aligned}
& \Delta P=P_{i}^{k+1}-P_{i}^{k}=\lambda_{7} \frac{\left(U_{i, P}^{k}-\overline{U_{P}^{k}}\right) P_{i}^{k}}{\overline{U_{P}^{k}}}+r P^{k}, \\
& \Delta E=E_{i}^{k+1}-E_{i}^{k}=\lambda_{8} \frac{\left(U_{i, E}^{k}-\overline{U_{E}^{k}}\right) E_{i}^{k}}{\overline{U_{E}^{k}}}+r E^{k} .
\end{aligned}
$$

3.7. The Multilayer Network Growth Process. The multilayer network coevolution process is combined with the coevolution model. The design purpose of the rail network is to serve more people and increase convenience. Hence, the first objective is to maximise the total population covered. The second objective is to minimise the total travel costs-or to obtain a better network structure. For the upper-layer, a new node is randomly selected from these lower-layer nodes which have the highest population, and a new scale-free network is generated. For the lower-layer, a random number of new nodes (less than 10) will be added in, and a new scalefree network is generated. The network user will choose to use the rail networks for saving travel cost, and then, based on the limited capacity of rail networks, it has the upgraded distribution of traffic flow. Based on this, the population will be redistributed.

\section{Solution Process and Related Simulation Scenarios}

To solve the model, this section proposed a solution process given here, and the related simulation scenarios are discussed.

4.1. Solution Process. Here is the solution process by steps:

Step 1. Set the initial amount of population and employment (different land-use situations) and the travel speeds, and distribute them in a square area with $100 \mathrm{~km}$ of side length and 100 nodes. Here, the random network is used to discuss the growth model, and the population is equal to the employment.

Step 2. Based on the functions of traffic demand model, at each step, it can have the initial OD matrix and the initial travel costs, and the traffic flows can be measured based on the traffic assignment model by User Equilibrium (UE) model and Frank-Wolfe algorithm and converge to $5 \%$ of estimation error range.

Step 3.The collected revenues and overall expenditure can be calculated by the traffic flows. Generally, consider the spending of the total profit for the network growth, and the multilayer network growth process introduced here. With the growth of multilayer networks, the network growth will be based on them with optimised APL, $D$, and travel cost. After the network growth and considering these flows on the rail networks, the free flow speed of new links, and link capacity, traffic flows on different links 
including upper-layers can be updated, and an equilibrium status can be reached again.

Step 4. Considering the change of population growth and network structures, land-use accessibility and desirability are changed, which causes the redistribution of population and employment, and then the OD matrix and traffic flows change. Then, with the new distribution of population and employment, the new travel speeds and network structure can be obtained; return to Step 1.

4.2. Related Simulation Scenarios. As per Step 3 and the initial status of the simulated city proposed, some scenarios will be considered here as in Table 1 . For simplicity, here, we merely tested the model with the regular network not growing, and then more focus was given to the multilayer network growth-related models. Here, this study considers 8 different simulation scenarios; for example, RRR stands for the initial population and employment, travel speeds are randomly distributed (see Figure 5), and so on. As the relationship between land-use and population density could be modelled well [48], the discussion of different land-use scenarios can turn to the discussion of the distribution of population and employment.

\section{Simulation and Validation}

In the same way as the simulated city, suppose that a square urban area with length equal to $10 \times 10^{4}$ meters has an initial number of nodes (randomly and regularly distributed when the network is not growing to test the proposed model, and randomly distributed when the network is growing) $(n=100)$, with an initial population of 500 thousand, and the natural increase rate of population is $1.5 \%$ per iteration. Then, the model validation part is proposed at the end of this section.

5.1. When the Network Is Not Growing. The total profits are used for the capacity increase of each line, which means that although the network is not growing, the capacities are improving. Because the network structure is not changed, it cannot measure the change of APL, D, EG, and so on; hence, the study will more focus on the total travel times which not only show how easily one citizen can arrive one area but also describe the traffic status of the whole network. With the initial traffic flows on each street, the total travel time cost through these congested streets is also shown in Figure 6. With iteration, we can see that this time cost of different simulation scenarios decreases sharply until reaching a stable state (Figure 7) (results come from 10 times of simulation), which means that the street capacity is optimised during the population redistribution process. No matter what kind of simulation scenarios the network initially chose, at last, they have similar trends. The population is redistributed following the network travel cost change, and some areas with higher population also emerge because of the change of street capacity and travel speed.
Here, the red lines are the travel times of each street, and the width stands for their values. The black lines are the traffic flows on these streets. Here is the performance of the random network and regular network in 6 iterations.

5.2. When the Network Is Growing. With the growth of networks, based on a random network shown in Figure 8, in line with previous researches, the change trends of APL and $D$ can be obtained. Considering these different scenarios, we can see that, with the increase of multilayer networks, the APL and $D$ are ultimately decreased. With the growth of the rail network, it is clear that more traffic flows are attracted by the rail network, and after a few steps, the traffic flow becomes more evenly distributed.

APL decreased from more than $5.9 \times 103$ to around $4 \times 103$, a decrease of about $32 \%$ (see Figure 9), and $D$ decreased from $15 \times 104$ to $8 \times 104$, a decrease of about $46 \%$ (see Figure 10). With the network growth process, the increase of network efficiency (Figure 11) by around 27\% to $44 \%$ can be seen, with the URU having the lowest change rate and RUU having the highest change rate, which means that, with the multilayer network growth model, the proposed network structure became optimised. The total traffic flow is optimised by around 30\% of decrease (Figure 12) by the process of redistribution of population and employment and land-use change, which reduce the movement between two nodes and make the population and employment reach the balance in an area, and intrazonal movement increases. Obviously, the total travel cost is also decreased; this also means the improvement of network efficiency. Some nodes with higher population have emerged with their advanced traffic accessibility, and "new town" or "developing area" also emerged, as more and more new nodes are located in the right middle area.

5.3. Model Validation. The change of average travel speed ratio can influence the model choice and the growth of networks [23]. To validate the simulation results, another quantitative index is proposed here as beta, the average travel speed ratio between upper-layer and lower-layer networks, and the function is

$$
\text { beta }=\frac{v_{a}^{k}}{v_{r}}, \quad \text { beta } \in(0,1),
$$

where $v_{a}^{k}$ is the average travel speed of the street network and $v_{r}$ is the average travel speed of the rail network. Here, we set the average travel speed of the rail network as a constant; then, as the value of beta increases from 0 to 1 , this means that the average travel speed of the street network is increasing.

Here, to validate the model, we have run the CNIMUGOM 100 times with UUU simulation scenario with the same network and have the average value of the APL (see Figure 13) and traffic flow (see Figure 14) with different beta values. It is shown that, with the value of beta belonging to 0.2 to 0.3 , the APL has the lowest value. While the situation changes a little bit when the traffic flow is considered, 
TABLE 1: The simulation scenarios.

\begin{tabular}{lccc}
\hline No. & The population distribution & The employment distribution & The limited street speeds \\
\hline Proposed models & Randomly (R)/uniformly (U) & Randomly (R)/uniformly (U) & Randomly (R)/uniformly (U) \\
1 & $\mathrm{R}$ & $\mathrm{R}$ & $\mathrm{R}$ \\
2 & $\mathrm{R}$ & $\mathrm{R}$ & $\mathrm{U}$ \\
3 & $\mathrm{R}$ & $\mathrm{U}$ & $\mathrm{R}$ \\
4 & $\mathrm{R}$ & $\mathrm{U}$ & $\mathrm{U}$ \\
5 & $\mathrm{U}$ & $\mathrm{R}$ & $\mathrm{R}$ \\
6 & $\mathrm{U}$ & $\mathrm{U}$ & $\mathrm{U}$ \\
7 & $\mathrm{U}$ & $\mathrm{U}$ & $\mathrm{R}$ \\
8 & $\mathrm{U}$ & & $\mathrm{U}$ \\
\hline
\end{tabular}

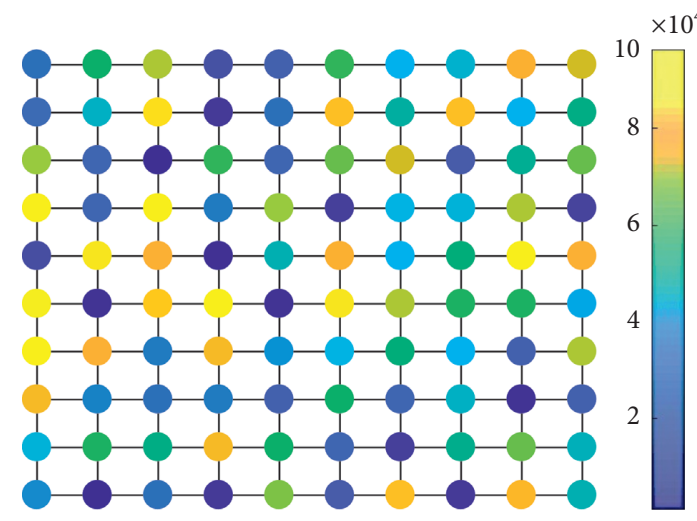

(a)

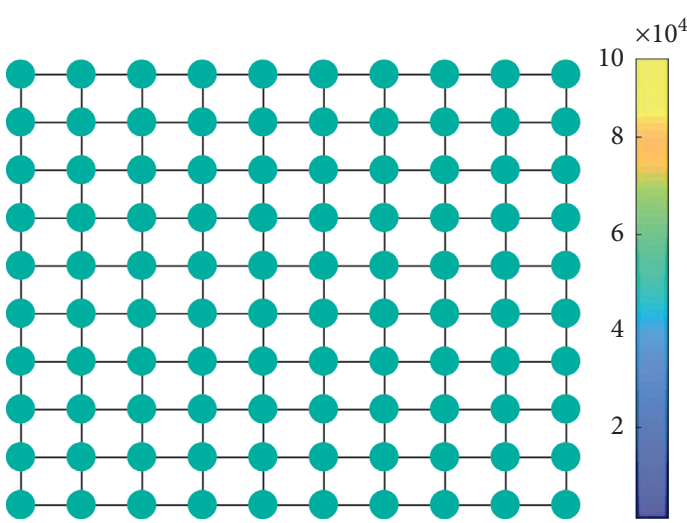

(c)

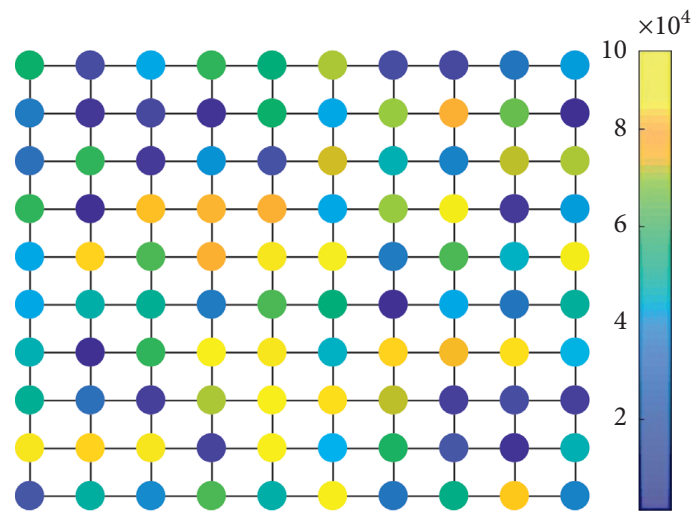

(b)

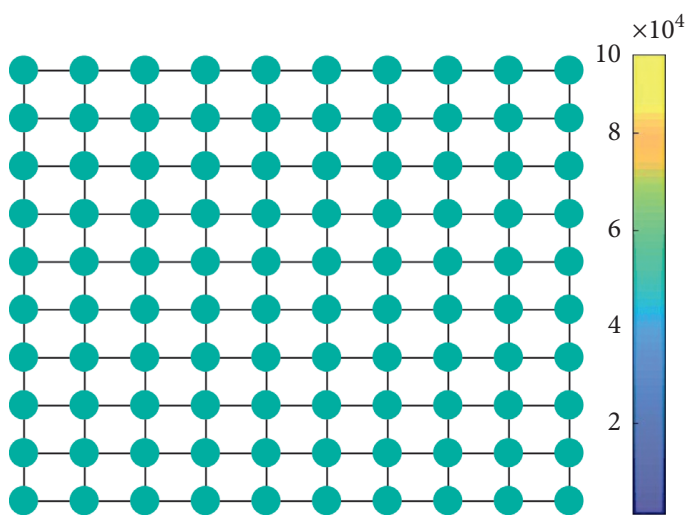

(d)

Figure 5: The initial distribution of population, employment, and travel speeds. Here, for each network, 100 nodes are plotted, and the black edges here stand for the travel speeds. (a) The population, employment, and travel speeds are randomly distributed. (b) The population and employment are randomly distributed, but the travel speeds are unified. (c) The population and employment are unified, but the travel speeds are randomly distributed. (d) All of them are uniformly distributed. The colour bar stands for the population amount, the colour of each node can stand for the population and employment amount, and the same is true for the multilayer network representation. 


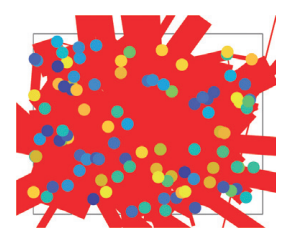

(a)

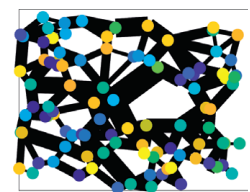

(g)

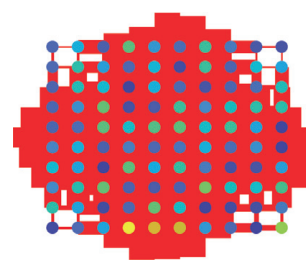

(m)

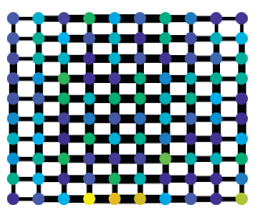

(s)

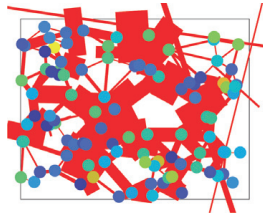

(b)

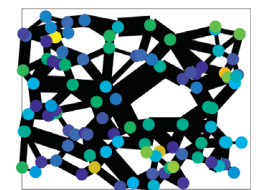

(h)

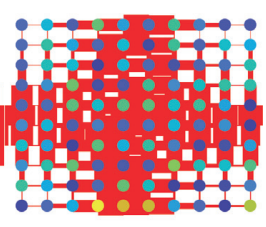

(n)

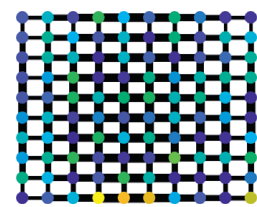

$(\mathrm{t})$

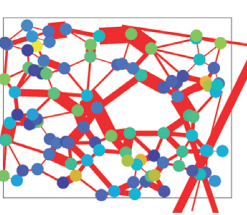

(c)

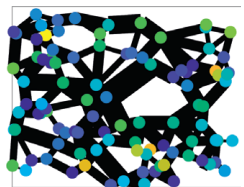

(i)

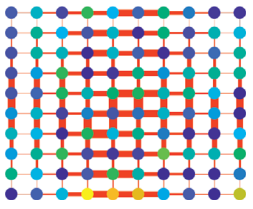

(o)

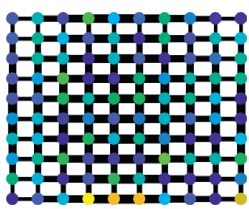

(u)

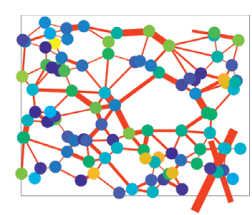

(d)

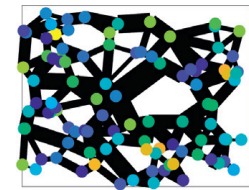

(j)

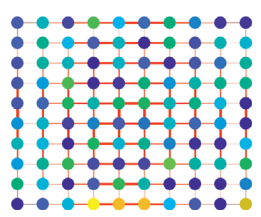

(p)

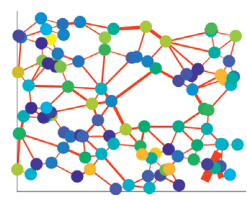

(e)

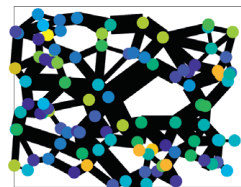

(k)

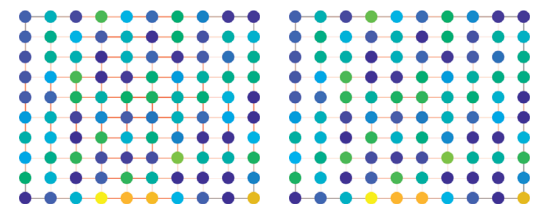

(q)

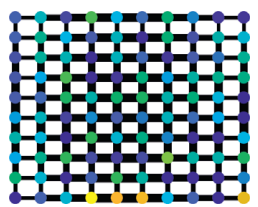

(v)

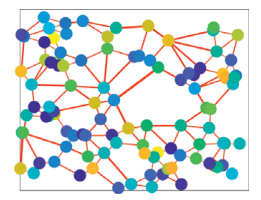

(f)

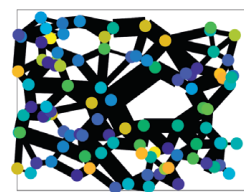

(1)

(r)

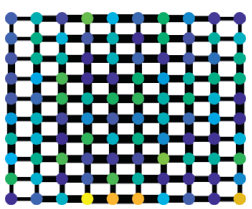

(w)

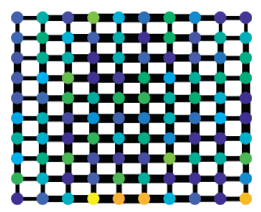

(x)

FIGURE 6: The optimisation of population distribution and travel times on the streets.

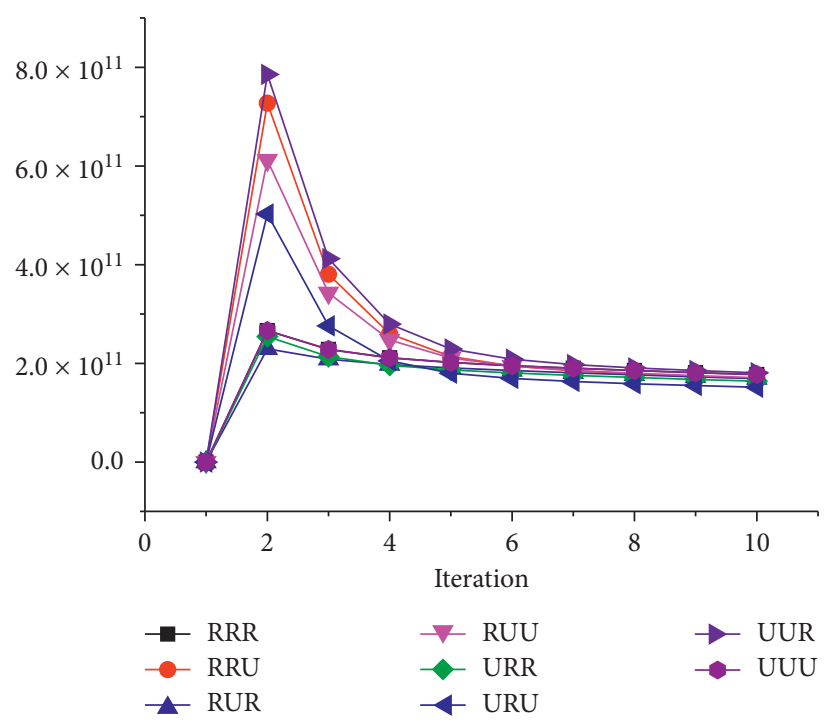

Figure 7: The total time cost of different simulation scenarios decreases sharply. 


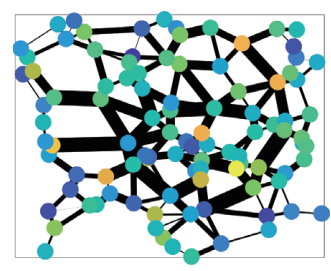

(a)

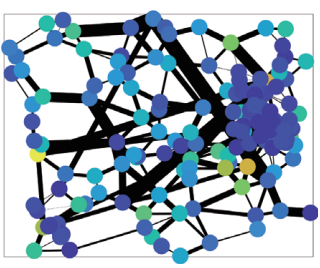

(f)

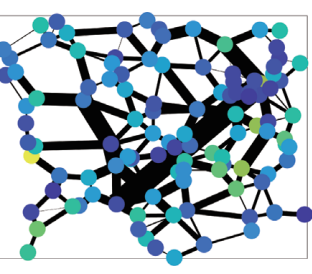

(b)

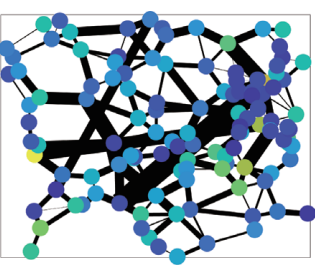

(c)

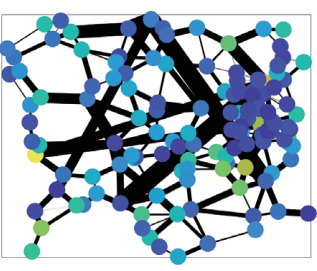

(d)

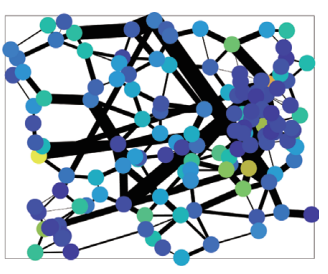

(e)

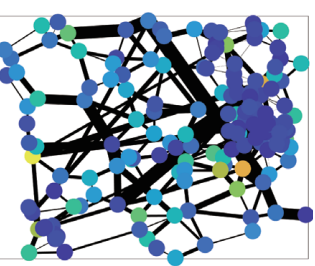

(g)

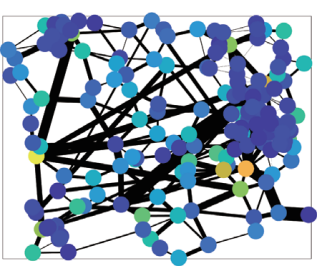

(h)

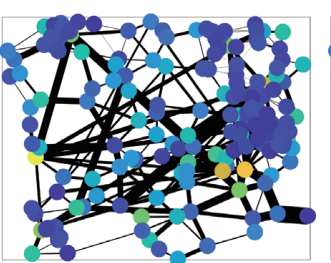

(i)

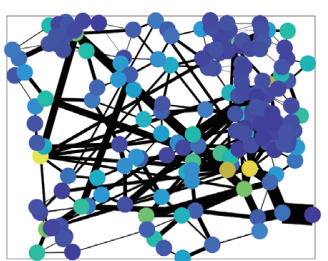

(j)

FiguRE 8: The growth of multilayer network and redistribution of population and traffic flow, increasing from the top left to bottom right with ten times of iterations.

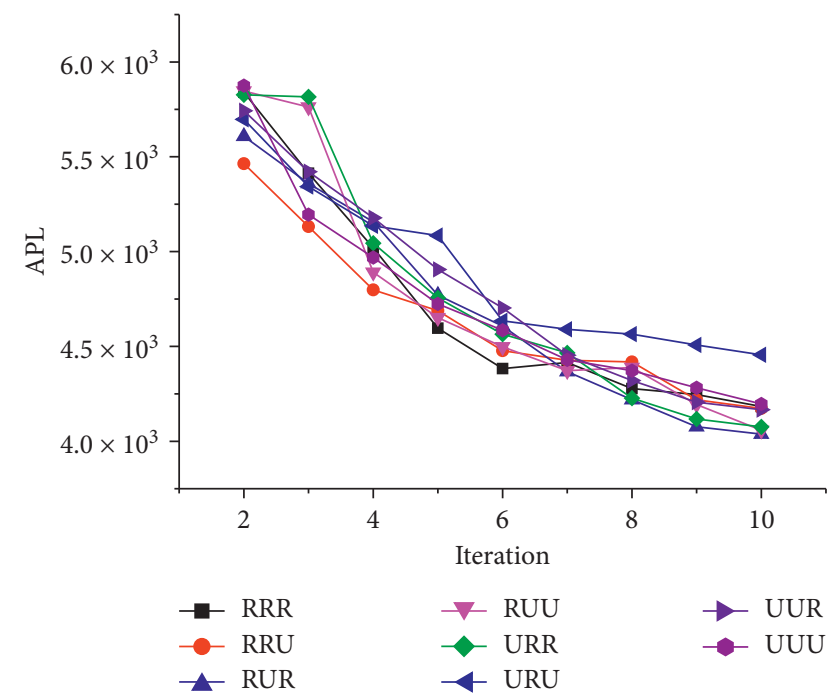

FIGURE 9: The change trends of APL of different simulation scenarios.

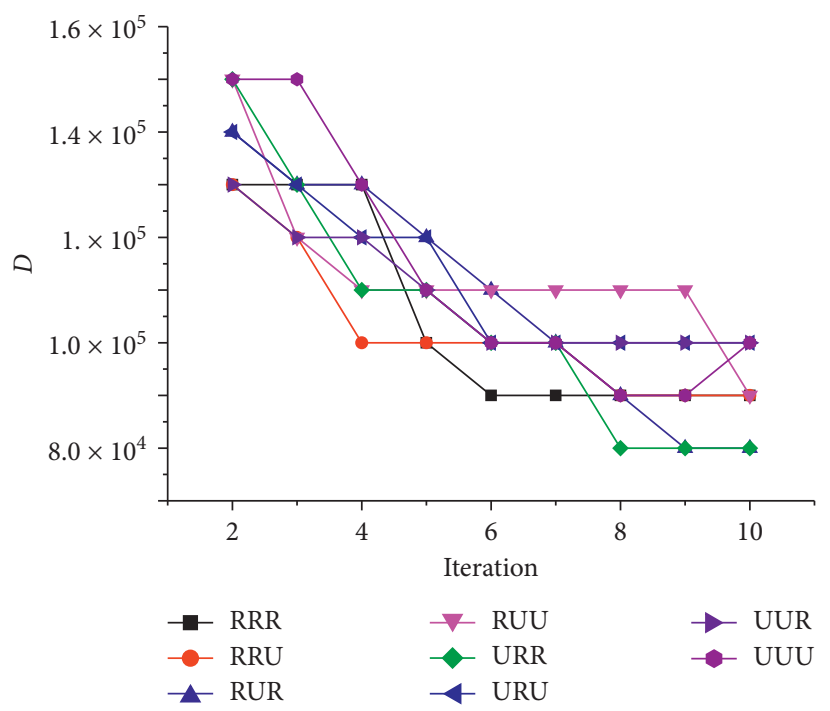

Figure 10: The change trends of $\mathrm{D}$ of different simulation scenarios.

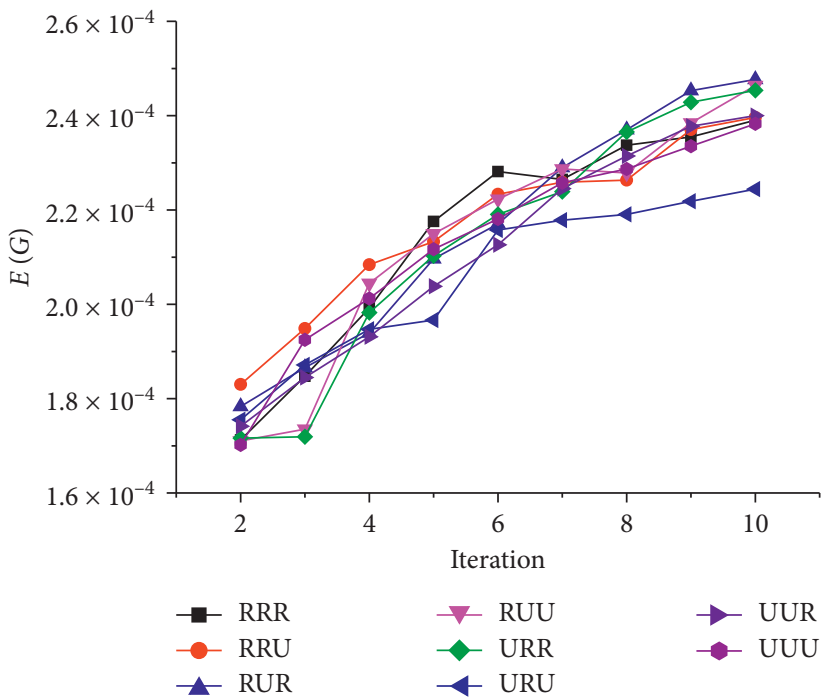

FIGURE 11: The change trends of network efficiency of different simulation scenarios.

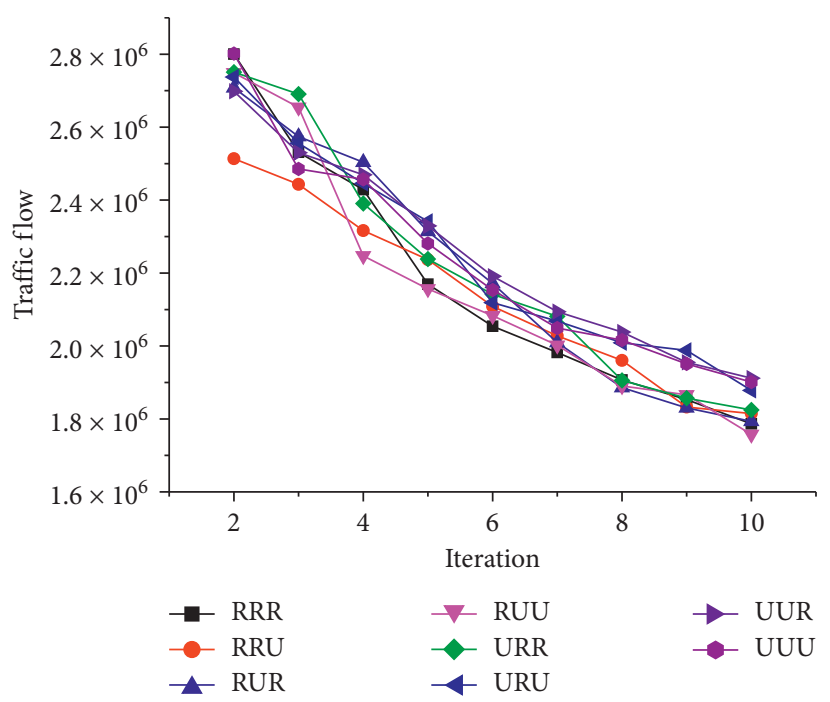

FIGURE 12: The change trends of traffic flow of different simulation scenarios. 


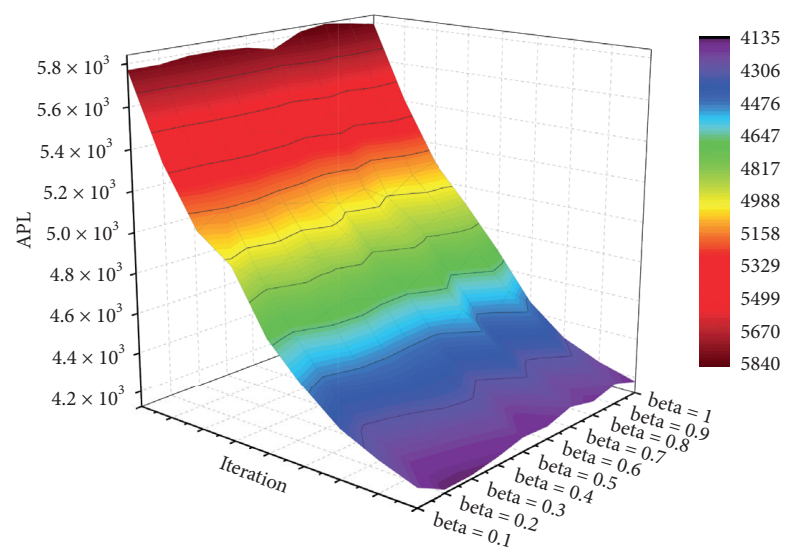

FIgURE 13: The change trends of APL with different beta values.

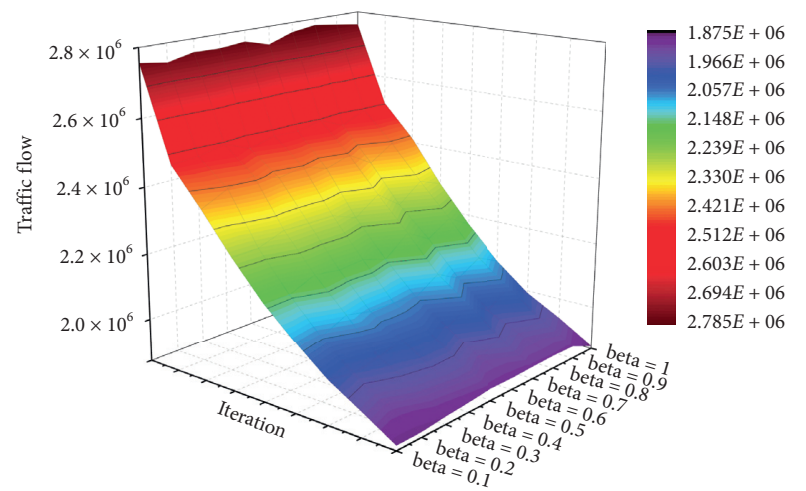

Figure 14: The change trends of traffic flow with different beta values.

showing that the traffic flow has the lowest value when the value of beta belongs to 0.1 to 0.2 . Furthermore, the CNIMUGOM works pretty stably with different beta values and simulation scenarios.

\section{Conclusion}

Research about coevolution models and multilayer network models remains somewhat superficial; the combinations of coevolution models and multilayer network models with land-use applications are relatively rare. While research in this area is resolutely forward-looking and fulfils the requirements of mathematical ability, as with previous city models, it lacks a systematic and comprehensive economic description and combination with urban economic theory.

The proposed CNIMUGOM has expanded the traditional planar model to a multilayer network model, which can better fit the real situations. This research also considered the multilayer network coevolution process further and considered the self-organisation properties of networks. This model has proposed an efficient way to combine these different traffic modes, well considering the complex evolution and coevolution relationships between network structure, land-use, and population.
CNIMUGOM can be used to optimise the APL and $D$, to make the network have smaller total traffic flow, and save the total travel cost; in other words, we can have a more efficient network structure. The inner relationships between submodels such as the travel demand model, street investment model, traffic network growth model, population growth model, urban land-use growth model, and network optimisation model were analysed and discussed.

The proposed CNIMUGOM has some superior properties compared to other models, as it is a universal model and can be used in many cities for their modelling optimal planning schemes and for the large-scale investments. However, some parts can be further improved. For example, the growth rate of the population is based on the exponential distribution for short-term analysis, which may not be very close to the real situation, and the logistic population growth model can be used for long-term analysis to adjust it. The study of economic models is still open and can be combined with big data; with the study of CNIMUGOM, the population distribution acquisition method of big data can be adopted; for example, the cellphone signalling data and GPS data can be used to represent the distribution of the urban population. Next, we proposed that all the revenues are used to improve the network structure and link capacity; however, in reality, the incomes can hardly be all used for the improvement of the networks. Thirdly, the redistribution of the population, for now, is relatively hard, which has to consider the place attachment for the population and the difficulty of its relocation, but from the experiences of Beijing, based on the public rental housing projects, it can be partly done. For now, the CNIMUGOM is based on the simulated city data; for a real city, the model can be easily applied with road network data, GPS data, and population data.

\section{Data Availability}

The simulated data used to support the findings of this study are included within the article, and related codes are available from the corresponding author upon request.

\section{Disclosure}

This paper is modified and expanded based on Section 4.4 of Rui Ding's doctoral dissertation.

\section{Conflicts of Interest}

The authors declare that they have no conflicts of interest.

\section{Acknowledgments}

This study was funded by National Natural Science Foundation of China (No. 72001053) and Science and Technology Planning Project of Guizhou Province of China (No. Qian ke he ji chu [2020]1Y283). Universiti Putra Malaysia is hereby acknowledged. 


\section{References}

[1] S. Abdullahi, B. Pradhan, and A. A. Al-Sharif, Introduction to Urban Growth and Expansion Spatial Modeling and Assessment of Urban Form, Springer, Berlin, Germany, 2017.

[2] M. Barthélemy and A. Flammini, "Co-evolution of density and topology in a simple model of city formation," Networks and Spatial Economics, vol. 9, no. 3, pp. 401-425, 2009.

[3] J. Wu, R. Li, R. Ding, T. Li, and H. Sun, "City expansion model based on population diffusion and road growth," Applied Mathematical Modelling, vol. 43, pp. 1-14, 2017.

[4] R. Ding, N. Ujang, H. Bin Hamid, and J. Wu, "Complex network theory applied to the growth of kuala lumpur's public urban rail transit network," PLoS One, vol. 10, no. 10, Article ID e0139961, 2015.

[5] F. Xie and D. Levinson, "Modeling the growth of transportation networks: a comprehensive review," Networks and Spatial Economics, vol. 9, no. 3, pp. 291-307, 2009.

[6] D. Yamins, S. Rasmussen, and D. Fogel, "Growing urban roads," Networks and Spatial Economics, vol. 3, no. 1, pp. 69-85, 2003.

[7] R. Ding, "The complex network theory-based urban land-use and transport interaction studies," Complexity, vol. 201914 pages, 2019.

[8] R. Ding, N. Ujang, H. B. Hamid et al., "Application of complex networks theory in urban traffic network researches," Networks and Spatial Economics, vol. 19, pp. 1-37, 2019.

[9] D. Levinson, "Density and dispersion: the co-development of land use and rail in London," Journal of Economic Geography, vol. 8, Article ID lbm038, 2007.

[10] F. Xie and D. Levinson, "Measuring the structure of road networks," Geographical Analysis, vol. 39, no. 3, pp. 336-356, 2007.

[11] F. Xie and D. Levinson, "Topological evolution of surface transportation networks," Computers, Environment and Urban Systems, vol. 33, no. 3, pp. 211-223, 2009.

[12] J. Wu, M. Xu, and Z. Gao, "Coevolution dynamics model of road surface and urban traffic structure," Nonlinear Dynamics, vol. 73, no. 3, pp. 1327-1334, 2013.

[13] J. Wu, M. Xu, and Z. Gao, "Modeling the coevolution of road expansion and urban traffic growth," Advances in Complex Systems, vol. 17, no. 1, Article ID 1450005, 2014.

[14] Y. Rui, Urban growth modeling based on land-use changes and road network expansion, , Ph.D. thesis, Stockholm, Sweden, 2013.

[15] T. Li, H. Sun, J. Wu, Z. Gao, Y.-E. Ge, and R. Ding, "Optimal urban expressway system in a transportation and land use interaction equilibrium framework," Transportmetrica A: Transport Science, vol. 15, no. 2, pp. 1247-1277, 2019.

[16] M. Kurant and P. Thiran, "Layered complex networks," Physical Review Letters, vol. 96, no. 13, Article ID 138701, 2006.

[17] J. Ma, W. Han, Q. Guo, and Z. Wang, "Traffic dynamics on two-layer complex networks with limited delivering capacity," Physica A: Statistical Mechanics and Its Applications, vol. 456, pp. 281-287, 2016.

[18] S.-R. Albert, G. Sergio, and A. Alex, "Congestion induced by the structure of multiplex networks," Physical Review Letters, vol. 116, no. 10, Article ID 108701, 2016.

[19] A. Aleta, S. Meloni, and Y. Moreno, "A multilayer perspective for the analysis of urban transportation systems," Nature, vol. 7, 2016.

[20] R. Ding, N. Ujang, H. B. Hamid, M. S. A. Manan, R. Li, and J. Wu, "Heuristic urban transportation network design method, a multilayer coevolution approach," Physica A: Statistical Mechanics and Its Applications, vol. 479, pp. 71-83, 2017.

[21] R. Gallotti, A. Bazzani, S. Rambaldi, and M. Barthelemy, "How transportation hierarchy shapes human mobility," 2015.

[22] R. G. Morris and M. Barthelemy, "Transport on coupled spatial networks," Physical Review Letters, vol. 109, no. 12, Article ID 128703, 2012.

[23] E. Strano, S. Shai, S. Dobson, and M. Barthelemy, "Multiplex networks in metropolitan areas: generic features and local effects," Journal of The Royal Society Interface, vol. 12, no. 111, Article ID 20150651, 2015.

[24] R. Ding, N. Ujang, H. B. Hamid et al., "Detecting the urban traffic network structure dynamics through the growth and analysis of multi-layer networks," Physica A: Statistical Mechanics and Its Applications, vol. 503, pp. 800-817, 2018.

[25] P. Crucitti, V. Latora, and S. Porta, "Centrality in networks of urban streets," Chaos: An Interdisciplinary Journal of Nonlinear Science, vol. 16, no. 1, Article ID 15113, 2006.

[26] B. Jiang and C. Claramunt, "Topological analysis of urban street networks," Environment and Planning B: Planning and Design, vol. 31, no. 1, pp. 151-162, 2004.

[27] V. Latora and M. Marchiori, "Is the Boston subway a smallworld network?" Physica A: Statistical Mechanics and Its Applications, vol. 314, no. 1-4, pp. 109-113, 2002.

[28] M. Newman, A.-L. Barabasi, and D. J. Watts, The Structure and Dynamics of Networks, Princeton University Press, Princeton, NJ, USA, 2011.

[29] L. C. Freeman, "A set of measures of centrality based on betweenness," Sociometry, vol. 40, no. 1, pp. 35-41, 1977.

[30] M. Marchiori and V. Latora, "Harmony in the small-world," Physica A: Statistical Mechanics and its Applications, vol. 285, no. 3, pp. 539-546, 2000.

[31] R. Albert and A.-L. Barabási, "Statistical mechanics of complex networks," Reviews of Modern Physics, vol. 74, no. 1, p. 47, 2002.

[32] V. Latora and M. Marchiori, "Efficient behavior of smallworld networks," Physical Review Letters, vol. 87, no. 19, Article ID 198701, 2001.

[33] C.-G. Gu, S.-R. Zou, X.-L. Xu et al., "Onset of cooperation between layered networks," Physical Review E, vol. 84, no. 2, Article ID 26101, 2011.

[34] H. Sun, Z. Gao, and J. Wu, "A bi-level programming model and solution algorithm for the location of logistics distribution centers," Applied Mathematical Modelling, vol. 32, no. 4, pp. 610-616, 2008.

[35] S. Boccaletti, V. Latora, Y. Moreno, M. Chavez, and D. Hwang, "Complex networks: structure and dynamics," Physics Reports, vol. 424, no. 4, pp. 175-308, 2006.

[36] H. Xiaoyan, Z. Shuang, and C. Xiaoshu, "Spatial-temporal evolution of Guangzhou subway accessibility and its effects on the accessibility of public transportation services," Progress in Geography, vol. 33, no. 8, pp. 1078-1089, 2014.

[37] M. Frank and P. Wolfe, "An algorithm for quadratic programming," Naval Research Logistics Quarterly, vol. 3, no. 1, pp. 95-110, 1956.

[38] M. Jaggi, "Revisiting frank-wolfe: projection-free sparse convex optimization," in Proceedings of the 30th International Conference on Machine Learning, Atlanta, GA, USA, 2013.

[39] C. F. Daganzo and Y. Sheffi, "On stochastic models of traffic assignment," Transportation Science, vol. 11, no. 3, pp. 253-274, 1977. 
[40] M. Fukushima, "A modified frank-wolfe algorithm for solving the traffic assignment problem," Transportation Research Part B: Methodological, vol. 18, no. 2, pp. 169-177, 1984.

[41] L. J. LeBlanc, R. V. Helgason, and D. E. Boyce, "Improved efficiency of the frank-wolfe algorithm for convex network programs," Transportation Science, vol. 19, no. 4, pp. 445-462, 1985.

[42] Y. Sheffi, Urban Transportation Networks, Prentice-Hall, Englewood Cliffs, NJ, USA, 1985.

[43] G. Giuliano, "Land use impacts of transportation investments," The Geography of Urban Transportation, pp. 237-273, Guilford Publications, New York, NY, USA, 2004.

[44] M. Wegener, "Overview of land use transport models," Handbook of Transport Geography and Spatial Systems, vol. 5, pp. 127-146, 2004.

[45] J. Gutiérrez, A. Monzón, and J. M. Piñero, "Accessibility, network efficiency, and transport infrastructure planning," Environment and Planning A: Economy and Space, vol. 30, no. 8, pp. 1337-1350, 1998.

[46] L. C. Molin Zhao and F. Li, "Railway network evaluation based on complex network analysis," Advances in Applied Mathematics, vol. 5, no. 3, pp. 560-566, 2016.

[47] L. Li, Y. Sato, and H. Zhu, "Simulating spatial urban expansion based on a physical process," Landscape and Urban Planning, vol. 64, no. 1, pp. 67-76, 2003.

[48] F. Li, S. Zhang, K. Bu, J. Yang, Q. Wang, and L. Chang, "The relationships between land use change and demographic dynamics in western Jilin province," Journal of Geographical Sciences, vol. 25, no. 5, pp. 617-636, 2015.

[49] F. Levinson Xie and S. Zhu, "The co-evolution of land use and road networks," Transportation and Traffic Theory, pp. 839859, 2007.

[50] T. Li, J. Wu, H. Sun, and Z. Gao, "Integrated co-evolution model of land use and traffic network design," Networks and Spatial Economics, vol. 16, no. 2, pp. 579-603, 2015.

[51] D. Sun, K. Zhang, and S. Shen, "Analyzing spatiotemporal traffic line source emissions based on massive didi online carhailing service data," Transportation Research Part D: Transport and Environment, vol. 62, pp. 699-714, 2018.

[52] J. Sun and X. Ding, "Spatiotemporal evolution of ridesourcing markets under the new restriction policy: a case study in Shanghai," Transportation Research Part A Policy and Practice, vol. 130, no. 227, p. 239, 2019.

[53] K. Zhang, D. J. Sun, S. Shen, and Y. Zhu, "Analyzing spatiotemporal congestion pattern on urban roads based on taxi GPS data," Journal of Transport \& Land Use, vol. 10, no. 1, pp. 675-694, 2017.

[54] F. Xie and D. Levinson, Evolving Transportation Networks, Springer Science \& Business Media, New York, NY, USA, 2011.

[55] M. Batty and S. Mackie, "The calibration of gravity, entropy, and related models of spatial interaction," Environment and Planning A: Economy and Space, vol. 4, no. 2, pp. 205-233, 1972.

[56] X. Xie, "Calibration method and comparison of gravity model," Commun Stand, vol. 8, pp. 17-20, 2008.

[57] J. De Dios Ortuzar and L. G. Willumsen, Modelling Transport, Wiley, Hoboken, NJ, USA, 1994.

[58] L. Zhang and D. Levinson, "Road pricing with autonomous links," Transportation Research Record: Journal of the Transportation Research Board, vol. 1932, no. 1, pp. 147-155, 2005.
[59] F. Zhao, J. Wu, H. Sun, Z. Gao, and R. Liu, "Population-driven urban road evolution dynamic model," Networks and Spatial Economics, vol. 16, no. 4, pp. 997-1018, 2015.

[60] F. Wang, "Urban population distribution with various road networks: a simulation approach," Environment and Planning B: Planning and Design, vol. 25, no. 2, pp. 265-278, 1998.

[61] W. Lutz, W. Sanderson, and S. Scherbov, "The coming acceleration of global population ageing," Nature, vol. 451, no. 7179, pp. 716-719, 2008.

[62] K. T. Geurs and B. Van Wee, "Accessibility evaluation of landuse and transport strategies: review and research directions," Journal of Transport Geography, vol. 12, no. 2, pp. 127-140, 2004. 